infection depended, for instance, on cell-cell contact with a limited number of virus-presenting cells (for example, dendritic cells; ref. 6) in the lymphoreticular system, productive viral infections would begin to occur at a very high rate as drug-resistant strains spread through the population of antigen-presenting cells, each of which infects a large number of $\mathrm{CD}^{+} \mathrm{T}$ cells. Once most virus-presenting cells in a lymphoid organ are infected, the kinetics of virus production will dominantly reflect the rate of replacement of infected $\mathrm{CD} 4^{+} \mathrm{T}$ cells by uninfected cells at the loci of effective contact with virus-presenting cells, and will occur at a much slower rate. Under such a scheme, limiting the spread of HIV-1 infection within the virus-presenting cell population would represent a promising approach to the therapy of HIV disease ${ }^{7}$.

Michael Bukrinsky

Kirk Manogue

Anthony Cerami

The Picower Institute for Medical

Research,

Manhasset, New York 11030, USA

1. Wain-Hobson, S. Nature 373, 102 (1995).

2. Coffin, J. Science 267, 483-5489 (1995).

3. Ho, D.D. et al. Nature 373, 123-126 (1995)

4. Wei, X. et al. Nature $373,117-122$ (1995).

4. Wei, X. et al. Nature $373,117-122(1995)$.
5. Malone, J. L., Simms, T.E., Gray, G.C., Wagner, K.F. \&

Malone, J. L., Simms, T.E., Gray, G.C., Wagr
Burge, J.R. J. AIDS 3, 144-151 (1990).

6. Pope, M. et al. Cell 78, 389-398 (1984)

7. Dubrovsky, L. et al. Molec. Med. 1, 217-230 (1995).

\section{Paradox remains}

SIR - The articles by Ho et al. ${ }^{1}$ and Wei et $a l{ }^{2}$ have been hailed as providing crucial new information that clarifies the enigma of HIV-mediated pathogenesis (see, for example, refs 3,4 ). To the extent that they have estimated equilibrium rate constants and provided an explanation for rapid development of drug resistance, these studies $^{1,2}$ do provide new and important information. But the central paradox of AIDS pathogenesis remains.

The new studies on the dynamics of HIV infection demonstrate that the underlying rate of virus production is still 4-20 times lower than the rate of cell turnover. Because each infected cell produces many virions, and a high proportion of them are known to be defective, there is about $100-1,000$-fold more cell death than can be accounted for by the observed rate of virus production ${ }^{5}$. It is a murder scene with far more bodies than bullets.

Instead of addressing this discrepancy, the authors" ${ }^{1,2}$ suggest that "virus must be underestimated", or that many more infected cells must be hiding in deep lymphoid organs where they cannot be observed. Taken at face value, however, these data demonstrate that the CD4 lymphocyte population is highly activated, and that the fate of uninfected cells may be much more important than that of infected cells in AIDS pathogenesis. This view is consistent with observations that the rate of spontaneous apoptosis in the peripheral lymphocytes of HIV-infected individuals is both sharply elevated and at least 10-100-fold higher than the frequency of productively infected cells ${ }^{6}$

Ho et al. suggest the analogy of a sink, with the tap and drain both equally wide open, which eventually empties because the "regenerative capacity of the immune system (the tap) is not infinite" and cannot quite keep up. However, the differential between the tap and drain is extremely small $\left(20-200 \times 10^{6}\right.$ cells per day) compared with the overall flow rate $\left(2 \times 10^{9}\right.$ cells per day), and must remain relatively fixed for an average of 10 years, as $\mathrm{CD} 4^{+}$ cell loss is roughly linear throughout most of the natural history of HIV infection? Further, this model would predict no CD4 loss until virus production exceeded a critical threshold, and then an accelerating cell loss as the virus burden increases.

A more plausible explanation for these data is that a mechanism that finely regulates CD4 replacement makes a slight error, resulting in the failure to completely replace the cells (infected and uninfected alike) which are lost through programmed cell death, the natural consequence of immune activation. We have argued that this is exactly what would be expected if the immune system were exposed to a persistent co-stimulatory $\mathrm{T}$ cell signal caused by the interaction of gpl20 with CD4 (ref. 8). This extra signal causes the control mechanism to sense an elevated state of immune responsiveness and downregulate the recovery of "memory" cells to prevent growth of the immune system $^{9}$. The result would be an inexorable but steady decay based on this difference in probability of survival.

Maddox ${ }^{4}$ wonders "why, after more than a decade of research, has it only now emerged that the response of the immune system to infection by HIV is hyperactivity rather than the opposite?". The answer to this question is that those who would see AIDS as a more-or-less conventional viral infection have consistently refused to recognize the paradoxes that are clearly evident in the experimental data. The problem continues.

Michael S. Ascher, Haynes W. Sheppard Russell W. Anderson, John F. Krowka

\section{Hans J. Bremermann}

Viral and Rickettsial Disease Laboratory, California Department of Health Services, Berkeley, California 94704, USA

\footnotetext{
1. Ho, D. D. et al. Nature 373, 123-126 (1995)

2. Wei, X. et al. Nature 373, 117-122 (1995).

3. Wain-Hobson, S. Nature 373, 102 (1995)

4. Wain-Hobson, S. Nature 373, 102 (1995

5. Sheppard, H. W. et al. Nature 364, 291 (1993).

5. Sheppard, H. W. et al. Nature 364, 291 (1993),
6. Gougeon, M. L. \& Montagnier, L. Science 260, 1269-1270 (1993).

7. Sheppard, H. W. et al. J. AIDS 7, 1159-1166 1993).

8. Ascher, M.S. \& Sheppard, H.W. J. AIOS 3, 177-191 (1990).

9. Sheppard, H. W. \& Ascher, M. S. J. AIDS 5, 143-147 (1992).
}

SIR - D. Ho et al. (Nature 373, 123-126; 1995) state that HIV decay slopes (clearance rate constants) are independent of the initial viral loads, and that the slopes do not correlate with the initial CD4 lymphocyte counts. They conclude that the viral decay slope is not dependent on the stage of HIV-1 infection (their Fig. $1 b$ ).

But regression analysis of the same data shows that the correlation between HIV RNA (copies per $\mathrm{ml}$ ) and the viral decay slopes is 0.17 , with a $P$ value of 0.47 . When we stratify study subjects' viral decay rates according to levels of HIV RNA with a cut-off point of $100(<100$ versus $\geqslant 100$ ), however, the viral decay rate in the stratum with lower RNA is significantly different from that in the stratum with higher RNA (Kruskal-Wallis test: $P=0.048$ ). The median of the viral decay rate in the lower RNA stratum was -0.28 , while the median in the higher RNA stratum was -0.38 . A univariate linear regression analysis shows that the dichotomous variable RNA $(<100$ versus $\geqslant 100$ ) was significantly associated with the viral decay rate $(P=0.026)$. These analyses suggest that when RNA copies were at higher levels, the viral decay slopes would be higher. In other words, the viral decay slope is a function of many factors, including initial viral load and the stage of HIV infection.

Ho et al. also state that the slopes are inversely correlated with the baseline CD4 counts. Exponential modelling of CD4 increases reveals that the slopes are inversely correlated with the initial CD4 lymphocyte counts; the authors conclude that the CD4 lymphocyte depletion seen in AIDS is primarily a consequence of the destruction of these cells induced by HIV-1, not a lack of their production. The increase in CD4 lymphocyte counts following ABT-538 administration was also modelled linearly and the authors claimed that although their two sets of analyses (modelling both exponential and linear increases) do not yield identical numerical results, they are in close agreement and emphasize the same qualitative points about HIV-1 pathogenesis.

Based on our univariate linear regression analysis, the slope of increase generated by an exponential growth model is significantly and inversely associated with the baseline CD4 counts $(P=0.01)$. Nevertheless, the slope of increase generated by a linear production model was not significantly correlated with the baseline CD4 $(P=0.17)$. After adjusting for the viral loads, measured by HIV RNA, both the linear and exponential slopes seem to be correlated with the baseline CD4 counts, but in opposite directions. With the slope obtained from a linear production model, the slope was, although borderline, significantly and positively associated with the baseline CD4, after controlling RNA levels $(P=0.06)$. This implies that the slope 